\title{
A GENERALIZATION OF MOORE-PENROSE BIORTHOGONAL SYSTEMS*
}

\author{
MASAYA MATSUURA ${ }^{\dagger}$
}

\begin{abstract}
In this paper, the notion of Moore-Penrose biorthogonal systems is generalized. In [Fiedler, Moore-Penrose biorthogonal systems in Euclidean spaces, Lin. Alg. Appl. 362 (2003), pp. 137-143], transformations of generating systems of Euclidean spaces are examined in connection with the Moore-Penrose inverses of their Gram matrices. In this paper, $g$-inverses are used instead of the Moore-Penrose inverses and, in particular, the details of transformations derived from reflexive $g$ inverses are studied. Furthermore, the characterization theorem of Moore-Penrose inverses in [Fiedler and Markham, A characterization of the Moore-Penrose inverse, Lin. Alg. Appl. 179 (1993), pp. 129-133] is extended to any reflexive $g$-inverse.
\end{abstract}

Key words. Generalized inverses, Moore-Penrose inverses, Biorthogonal systems.

AMS subject classifications. 15A03, 15A09.

1. Introduction. In [3], transformations of generating systems of Euclidean spaces are introduced based on the Moore-Penrose inverses of their Gram matrices in order to generalize the notion of biorthogonal systems. As a further generalization, we shall examine in this paper transformations of ordered systems of vectors based on any type of generalized inverse.

Before describing our results in detail, we shall introduce notation and terminology. Throughout this paper, all matrices are real and all inner product spaces are defined over $\mathbb{R}$. We note that the extension to the complex case is straightforward. We denote by $I_{n}$ the identity matrix of order $n$. If its dimension is obvious, we simply denote it by $I$. The $m \times n$ zero matrix is denoted by $O_{m, n}$ or simply by $O$. The symbol $G L(n)$ indicates the set of all $n \times n$ invertible matrices. Let $W$ be any inner product space and let $U=\left(u_{1}, u_{2}, \ldots, u_{m}\right)$ be any ordered system of vectors in $W$, that is, $u_{i} \in W(1 \leq i \leq m)$. By $\operatorname{rank} U$, we mean the dimension of the subspace of $W$ generated by $u_{1}, u_{2}, \ldots, u_{m}$. For any $m \times n$ matrix $A=\left[a_{i j}\right]$, we denote by $U A$ the ordered system of vectors in $W$ whose $j$ th vector is $\sum_{i=1}^{m} a_{i j} u_{i}(1 \leq j \leq n)$. For two ordered systems of vectors $U=\left(u_{1}, u_{2}, \ldots, u_{m}\right)$ and $V=\left(v_{1}, v_{2}, \ldots, v_{n}\right)$ in $W$, the symbol $G(U, V)$ denotes the $m \times n$ matrix whose $(i, j)$ th element is given by the inner product $\left\langle u_{i}, v_{j}\right\rangle$. Especially we put $G(U)=G(U, U)$. Note that $G(U)$ is the Gram matrix of $U$. Therefore $G(U) \geq 0$ and $\operatorname{rank} G(U)=\operatorname{rank} U$. For any $m \times k$ matrix $A$ and $n \times l$ matrix $B$, we can easily verify that $G(U A, V B)=G(V B, U A)^{\mathrm{T}}=A^{\mathrm{T}} G(U, V) B$. Let the systems $U$ and $V$ be given by $U=F C$ and $V=F D$, where $F=\left(f_{1}, f_{2}, \ldots, f_{d}\right)$ is an orthonormal base of $W, C$ is a $d \times m$ matrix and $D$ is a $d \times n$ matrix. Then $\operatorname{rank} U=\operatorname{rank} C, \operatorname{rank} V=\operatorname{rank} D$ and $G(U, V)=C^{\mathrm{T}} D$. Thus, by using well-known

* Received by the editors on 13 February 2003. Accepted for publication on 21 May 2003. Handling Editor: Miroslav Fiedler.

${ }^{\dagger}$ Department of Mathematical Informatics, Graduate School of Information Science and Technology, University of Tokyo, Bunkyo-ku, Tokyo, 113-8656, Japan (masaya@mist.i.u-tokyo.ac.jp). 
inequalities on the ranks of matrix products, we know

$$
\operatorname{rank} U+\operatorname{rank} V-\operatorname{dim} W \leq \operatorname{rank} G(U, V) \leq \min \{\operatorname{rank} U, \operatorname{rank} V\} .
$$

Especially $\operatorname{rank} G(U, V)=\operatorname{dim} W$ if and only if $\operatorname{rank} U=\operatorname{rank} V=\operatorname{dim} W$.

We shall review the fundamental facts on generalized inverses ( $g$-inverses) which are needed in later discussion (see e.g. [1]). Let $A$ be any $m \times n$ matrix. An $n \times m$ matrix $A^{-}$is a $g$-inverse of $A$ if and only if $A A^{-} A=A$. A $g$-inverse $A_{r}^{-}$of $A$ is called a reflexive $g$-inverse if $A_{r}^{-} A A_{r}^{-}=A_{r}^{-}$, which is equivalent to $\operatorname{rank} A_{r}^{-}=\operatorname{rank} A$. If $A$ is nonnegative definite, any symmetric reflexive $g$-inverse of $A$ is also nonnegative definite. A $g$-inverse $A_{m}^{-}$of $A$ is a minimum norm $g$-inverse if and only if $A_{m}^{-} A$ is symmetric. Moreover, a $g$-inverse $A_{l}^{-}$of $A$ is a least squares $g$-inverse if and only if $A A_{l}^{-}$is symmetric. Finally, there exists uniquely an $g$-inverse $A^{+}$, which is called the Moore-Penrose inverse, that satisfy all the properties mentioned above, that is, $A^{+} A A^{+}=A^{+},\left(A^{+} A\right)^{\mathrm{T}}=A^{+} A$ and $\left(A A^{+}\right)^{\mathrm{T}}=A A^{+}$. Let

$$
A=P\left[\begin{array}{cc}
A_{1} & O \\
O & O
\end{array}\right] Q^{\mathrm{T}},
$$

where $P$ and $Q$ are orthogonal matrices and $A_{1}$ is an invertible matrix. Then the Moore-Penrose inverse of $A$ is given by

$$
A^{+}=Q\left[\begin{array}{cc}
A_{1}^{-1} & O \\
O & O
\end{array}\right] P^{\mathrm{T}} .
$$

We now review the following fact, which immediately follows from Theorem 2.1 in [3] (see also Theorem 1 in [2]).

THEOREM 1.1. Let $U=\left(u_{1}, u_{2}, \ldots, u_{m}\right)$ be any ordered generating system of an inner product space $W$. Moreover, let $G(U)^{+}$be the Moore-Penrose inverse of $G(U)$. Then there exists uniquely an ordered system of vectors $\widetilde{U}=\left(\widetilde{u}_{1}, \widetilde{u}_{2}, \ldots, \widetilde{u}_{m}\right)$ in $W$ such that

$$
\begin{aligned}
& G(\widetilde{U})=G(U)^{+}, \\
& G(U, \widetilde{U})=G(U) G(U)^{+} .
\end{aligned}
$$

We shall give the following definition, which is equivalent to Definition 2.2 in [3].

Definition 1.2. We call the system $\widetilde{U}$ in Theorem 1.1 the Moore-Penrose biorthogonal system to $U$.

The next section is devoted to an extension of the above theorem and definition. Given two ordered systems of vectors $U=\left(u_{1}, u_{2}, \ldots, u_{m}\right)$ and $V=\left(v_{1}, v_{2}, \ldots, v_{n}\right)$ in an inner product space, we shall construct another pair of ordered systems $\widetilde{U}=$ $\left(\widetilde{u}_{1}, \widetilde{u}_{2}, \ldots, \widetilde{u}_{n}\right)$ and $\widetilde{V}=\left(\widetilde{v}_{1}, \widetilde{v}_{2}, \ldots, \widetilde{v}_{m}\right)$ based on an arbitrary $g$-inverse $G(U, V)^{-}$of the matrix $G(U, V)$. Particularly, we shall closely study the case where $G(U, V)^{-}$is a reflexive $g$-inverse.

In section 3, the characterization theorem of Moore-Penrose inverses in [2], which was used in [3], is extended to that of any reflexive $g$-inverses. 
2. $G$-inverse pairs. As a generalization of Theorem 1.1, we shall state the following theorem.

TheOREM 2.1. Let $U=\left(u_{1}, u_{2}, \ldots, u_{m}\right)$ and $V=\left(v_{1}, v_{2}, \ldots, v_{n}\right)$ be any ordered systems of vectors in an inner product space $W$. Moreover, let $G(U, V)^{-}$be any $g$ inverse of $G(U, V)$. Then there exist ordered systems of vectors $\widetilde{U}=\left(\widetilde{u}_{1}, \widetilde{u}_{2}, \ldots, \widetilde{u}_{n}\right)$ and $\widetilde{V}=\left(\widetilde{v}_{1}, \widetilde{v}_{2}, \ldots, \widetilde{v}_{m}\right)$ in $W$ such that

$$
\begin{aligned}
& G(\widetilde{U}, \widetilde{V})=G(U, V)^{-}, \\
& G(U, \widetilde{V})=G(U, V) G(U, V)^{-}, \\
& G(\widetilde{U}, V)=G(U, V)^{-} G(U, V),
\end{aligned}
$$

if and only if

$$
\operatorname{rank} G(U, V)^{-} \leq \operatorname{dim} W+2 \operatorname{rank} G(U, V)-\operatorname{rank} U-\operatorname{rank} V .
$$

REMARK 2.2. We know from (1.1) that if $G(U, V)^{-}$is a reflexive $g$-inverse of $G(U, V)$, then condition (2.4) is satisfied.

To prove the theorem above, we shall show the following lemma.

Lemma 2.3. Let $A$ be any $m \times d$ matrix, $B$ be any $d \times n$ matrix and $C$ be any $n \times m$ matrix. Then, there exist an $n \times d$ matrix $J$ and $a d \times m$ matrix $K$ such that

$$
J K=C, \quad J B=O, \quad A K=O,
$$

if and only if

$$
\operatorname{rank} A+\operatorname{rank} B+\operatorname{rank} C \leq d+\operatorname{rank} A B
$$

Proof. Let $\operatorname{rank} A=r_{A}, \operatorname{rank} B=r_{B}$ and $\operatorname{rank} A B=r_{A B}$. Then, there exists an invertible matrix $P$ such that

$$
A=\left[\begin{array}{ll}
A_{1} & O
\end{array}\right] P, \quad B=P^{-1}\left[\begin{array}{c}
B_{1} \\
O \\
B_{2}
\end{array}\right]
$$

where $A_{1}$ is an $m \times r_{A}$ matrix, $B_{1}$ is an $r_{A B} \times n$ matrix and $B_{2}$ is an $\left(d-r_{A}\right) \times n$ matrix. Furthermore, there exist a $\left(d-r_{A}\right) \times r_{A}$ matrix $Q_{1}$ and a $\left(d-r_{A}\right) \times\left(d-r_{A}\right)$ invertible matrix $Q_{2}$ such that

$$
\left[\begin{array}{cc}
I & O \\
Q_{1} & Q_{2}
\end{array}\right] P B=\left[\begin{array}{c}
B_{1} \\
O_{\left(r_{A}-r_{A B}\right), n} \\
B_{3} \\
O
\end{array}\right],
$$

where $B_{3}$ is an $\left(r_{B}-r_{A B}\right) \times n$ matrix. Hence $J B=O$ and $A K=O$ if and only if $J$ and $K$ are in the forms

$$
J=\left[\begin{array}{llll}
O_{n, r_{A B}} & J_{1} & O & J_{2}
\end{array}\right]\left[\begin{array}{cc}
I & O \\
Q_{1} & Q_{2}
\end{array}\right] P, \quad K=P^{-1}\left[\begin{array}{c}
O \\
K_{1}
\end{array}\right],
$$


where $J_{1}$ is an $n \times\left(r_{A}-r_{A B}\right)$ matrix, $J_{2}$ is an $n \times\left(d-r_{A}-r_{B}+r_{A B}\right)$ matrix and $K_{1}$ is an $\left(d-r_{A}\right) \times m$ matrix. In this case, $J K$ is expressed as

$$
J K=\left[\begin{array}{ll}
O & J_{2}
\end{array}\right] Q_{2} K_{1} .
$$

Thus an $n \times m$ matrix $C$ can be represented as $C=J K$ if and only if $\operatorname{rank} C \leq$ $d-r_{A}-r_{B}+r_{A B}$. Therefore we have the proof. $\square$

Proof of Theorem 2.1. We define a matrix $G$ by

$$
G=\left[\begin{array}{cc}
G(U, V) & G(U, V) G(U, V)^{-} \\
G(U, V)^{-} G(U, V) & G(U, V)^{-}
\end{array}\right] .
$$

Then we have

$$
\begin{aligned}
& {\left[\begin{array}{cc}
I & -G(U, V) \\
O & I
\end{array}\right] G\left[\begin{array}{cc}
I & O \\
-G(U, V) & I
\end{array}\right]=\left[\begin{array}{cc}
O & O \\
O & G(U, V)^{-}
\end{array}\right]} \\
& {\left[\begin{array}{cc}
I & O \\
-G(U, V)^{-} & I
\end{array}\right] G\left[\begin{array}{cc}
I & -G(U, V)^{-} \\
O & I
\end{array}\right]=\left[\begin{array}{cc}
G(U, V) & O \\
O & C
\end{array}\right],}
\end{aligned}
$$

where

$$
C=G(U, V)^{-}-G(U, V)^{-} G(U, V) G(U, V)^{-} .
$$

Hence

$$
\operatorname{rank} C=\operatorname{rank} G(U, V)^{-}-\operatorname{rank} G(U, V) .
$$

On the other hand, let $F=\left(f_{1}, f_{2}, \ldots, f_{d}\right)$ be an orthonormal base of $W$. Then, there exist an $m \times d$ matrix $A$ and a $d \times n$ matrix $B$ such that $U=F A^{\mathrm{T}}$ and $V=F B$. We note that

$$
\operatorname{rank} A=\operatorname{rank} U, \quad \operatorname{rank} B=\operatorname{rank} V, \quad A B=G(U, V) .
$$

We assume condition (2.4) is satisfied. Then condition (2.6) is also satisfied. Hence we know from Lemma 2.3 that there exist an $n \times d$ matrix $J$ and a $d \times m$ matrix $K$ that satisfy $(2.5)$. Therefore, we can define a pair of systems $(\widetilde{U}, \widetilde{V})$ which satisfies conditions (2.1), (2.2) and (2.3) by

$(2.7) \widetilde{U}=F\left[\begin{array}{ll}A^{\mathrm{T}} & J^{\mathrm{T}}\end{array}\right]\left[\begin{array}{c}\left(G(U, V)^{-}\right)^{\mathrm{T}} \\ I\end{array}\right], \quad \widetilde{V}=F\left[\begin{array}{ll}B & K\end{array}\right]\left[\begin{array}{c}G(U, V)^{-} \\ I\end{array}\right]$.

To show the converse, we assume that there exists a pair of systems $(\widetilde{U}, \widetilde{V})$ that satisfies conditions (2.1), (2.2) and (2.3). We know that there exist an $n \times d$ matrix $\widetilde{J}$ and a $d \times m$ matrix $\widetilde{K}$ such that $\widetilde{U}=F \widetilde{J}^{\mathrm{T}}$ and $\widetilde{V}=F \widetilde{K}$. Then, from conditions (2.1), (2.2) and (2.3), we have

$$
\widetilde{J} \widetilde{K}=G(U, V)^{-}, \quad A \widetilde{K}=G(U, V) G(U, V)^{-}, \quad \widetilde{J} B=G(U, V)^{-} G(U, V) .
$$


We define an $n \times d$ matrix $J$ and a $d \times m$ matrix $K$ by

$$
J=\left[\begin{array}{ll}
-G(U, V)^{-} & I
\end{array}\right]\left[\begin{array}{c}
A \\
\widetilde{J}
\end{array}\right], \quad K=\left[\begin{array}{ll}
B & \widetilde{K}
\end{array}\right]\left[\begin{array}{c}
-G(U, V)^{-} \\
I
\end{array}\right] .
$$

Then, $J$ and $K$ satisfy condition (2.5), which means (2.6). Thus we have (2.4).

We now introduce the notion of $g$-inverse pairs as a generalization of Definition 1.2 .

DEFinition 2.4. We call any pair of systems $(\widetilde{U}, \widetilde{V})$ that satisfies conditions (2.1), (2.2) and (2.3) a $g$-inverse pair to $(U, V)$ associated with $G(U, V)^{-}$.

In general, for a given pair of systems $(U, V)$ and a $g$-inverse $G(U, V)^{-}$, a $g$-inverse pair $(\widetilde{U}, \widetilde{V})$ is not uniquely determined. However, if $U$ and $V$ are both generating systems of $W$ and $G(U, V)^{-}$is a reflexive $g$-inverse of $G(U, V)$, then a $g$-inverse pair is uniquely determined, that is, we have the following theorem.

TheOREM 2.5. Let $U=\left(u_{1}, u_{2}, \ldots, u_{m}\right)$ and $V=\left(v_{1}, v_{2}, \ldots, v_{n}\right)$ be any ordered generating systems of an inner product space $W$. Moreover, let $G(U, V)_{r}^{-}$be any reflexive g-inverse of $G(U, V)$. Then there exists uniquely in $W$ a $g$-inverse pair $(\widetilde{U}, \widetilde{V})$ to $(U, V)$ associated with $G(U, V)_{r}^{-}$. Furthermore, both $\widetilde{U}$ and $\widetilde{V}$ are generating systems of $W$.

Proof. The existence of a $g$-inverse pair $(\widetilde{U}, \widetilde{V})$ follows from Remark 2.2. To show the uniqueness, let $F=\left(f_{1}, f_{2}, \ldots, f_{d}\right)$ be an orthonormal base of $W$. Moreover, let $A$ and $B$ be the matrices that satisfy $U=F A^{\mathrm{T}}$ and $V=F B$. We note $\operatorname{rank} A=$ $\operatorname{rank} B=d$. Therefore, condition (2.5) implies $J=O$ and $K=O$. Thus, it follows from (2.8) that $\widetilde{U}=F \widetilde{J}^{\mathrm{T}}$ and $\widetilde{V}=F \widetilde{K}$ are the only systems for which conditions (2.1), (2.2) and (2.3) hold, where

$$
\widetilde{J}=G(U, V)_{r}^{-} A, \quad \widetilde{K}=B G(U, V)_{r}^{-} .
$$

Furthermore, since $\operatorname{rank} G(\widetilde{U}, \widetilde{V})=\operatorname{rank} G(U, V)_{r}^{-}=\operatorname{rank} G(U, V)=\operatorname{dim} W$, we obtain $\operatorname{rank} \widetilde{U}=\operatorname{rank} \widetilde{V}=\operatorname{dim} W$. This means both $\widetilde{U}$ and $\widetilde{V}$ generate $W$.

REMARK 2.6. If we can choose $J \neq O$ or $K \neq O$ in (2.7), then $(\widetilde{U}, \widetilde{V})$ defined by replacing $J$ and $K$ with $2 J$ and $(1 / 2) K$ becomes another $g$-inverse pair. Therefore $(\widetilde{U}, \widetilde{V})$ is not unique. From this fact, we know that the condition assumed in Theorem 2.5 is weakest in asserting uniqueness.

Next, we shall investigate the case where $U=V$ in Theorem 2.1.

THEOREM 2.7. Let $U=\left(u_{1}, u_{2}, \ldots, u_{m}\right)$ be any ordered system of vectors in an inner product space $W$. Moreover, let $G(U)^{-}$be any $g$-inverse of $G(U)$. Then there exists an ordered system of vectors $\widetilde{U}=\left(\widetilde{u}_{1}, \widetilde{u}_{2}, \ldots, \widetilde{u}_{m}\right)$ in $W$ such that

$$
\begin{aligned}
& G(\tilde{U})=G(U)^{-}, \\
& G(U, \widetilde{U})=G(U) G(U)^{-},
\end{aligned}
$$

if and only if $G(U)^{-}$is nonnegative definite and

$$
\operatorname{rank} G(U)^{-} \leq \operatorname{dim} W
$$


For the proof of Theorem 2.7, we state the following lemma, which can be easily verified.

Lemma 2.8. Let $A$ be any $m \times d$ matrix and $C$ be any $m \times m$ nonnegative definite matrix. Then, there exists an $m \times d$ matrix $J$ such that

$$
J J^{\mathrm{T}}=C, \quad A J^{\mathrm{T}}=O,
$$

if and only if

$$
\operatorname{rank} A+\operatorname{rank} C \leq d
$$

Proof of Theorem 2.7. We can use the same method as in the proof of Theorem 2.1 by putting $V=U$. In this case, condition (2.11) is equivalent to (2.4). Assume that $G(U)^{-}$is nonnegative definite and satisfies condition (2.11). Then, by virtue of Lemma 2.8 , we can choose $J$ and $K$ so that $K=J^{\mathrm{T}}$ in (2.7), that is, $\widetilde{U}=\widetilde{V}$. The converse is obvious from Theorem $2.1 \mathrm{\square}$

Definition 2.9. We call any system $\widetilde{U}$ that satisfies conditions (2.9) and (2.10) a $g$-inverse system to $U$ associated with $G(U)^{-}$.

Combining Theorems 2.5 and 2.7, we obtain the following corollary.

Corollary 2.10. Let $U=\left(u_{1}, u_{2}, \ldots, u_{m}\right)$ be any ordered generating system of an inner product space $W$. Moreover, let $G(U)_{r}^{-}$be any symmetric reflexive g-inverse of $G(U)$. Then there exists uniquely in $W$ a g-inverse system $\widetilde{U}$ to $U$ associated with $G(U)_{r}^{-}$. Moreover, $\widetilde{U}$ is a generating system of $W$.

Proof. Since $G(U)$ is nonnegative definite, so is $G(U)_{r}^{-}$. Furthermore, we have $\operatorname{rank} G(U)_{r}^{-}=\operatorname{rank} G(U)=\operatorname{dim} W$. Hence, we can apply Theorem 2.7 together with Theorem 2.5. $\mathrm{\square}$

At the end of this section, we shall examine $g$-inverse pairs associated with reflexive least squares $g$-inverses and reflexive minimum norm $g$-inverses.

THEOREM 2.11. Let $U=\left(u_{1}, u_{2}, \ldots, u_{m}\right)$ and $V=\left(v_{1}, v_{2}, \ldots, v_{n}\right)$ be any ordered generating systems of an inner product space $W$. Then we have the following claims.

(i) Let $G(U, V)_{l}^{-}$be any reflexive least squares $g$-inverse of $G(U, V)$ and let $(\widetilde{U}, \widetilde{V})$ be the g-inverse pair to $(U, V)$ associated with $G(U, V)_{l}^{-}$. Then for any $m \times 1$ matrix $A, \widetilde{V} A=O$ if and only if $U A=O$.

(ii) Let $G(U, V)_{m}^{-}$be any reflexive minimum norm $g$-inverse of $G(U, V)$ and let $(\widetilde{U}, \widetilde{V})$ be the g-inverse pair to $(U, V)$ associated with $G(U, V)_{m}^{-}$. Then for any $n \times 1$ matrix $A, \widetilde{U} A=O$ if and only if $V A=O$.

Proof. We only prove (i) because (ii) is similarly proved. Since $G(U, V)_{l}^{-}$is a least squares $g$-inverse, we know from $(2.2)$ that $G(U, \widetilde{V})=G(\widetilde{V}, U)$. Therefore for any $m \times 1$ matrix $A$, we have $G(U, \widetilde{V} A)=G(\widetilde{V}, U A)$. Moreover we know from Theorem 2.5, $U$ and $\widetilde{V}$ are both generating systems of $W$. Hence, $\widetilde{V} A=O$ if and only if $U A=O$. $\mathrm{U}$

The above property is mentioned in [3] for Moore-Penrose biorthogonal systems. 
3. Characterization of reflexive $g$-inverses. The aim of this section is to generalize Theorem 1 in [2] and Theorem A in [3], which characterize Moore-Penrose inverses. The following theorem can be seen as an extension of Theorem 1 in [2].

THEOREM 3.1. Let $A$ be any $m \times n$ matrix.

(i) Let $X$ be any $n \times n$ matrix and $Y$ be any $m \times m$ matrix such that

$$
\begin{array}{ll}
A X=A, & \operatorname{rank} X=\operatorname{rank} A, \\
Y A=A, & \operatorname{rank} Y=\operatorname{rank} A .
\end{array}
$$

Then, an $n \times m$ matrix $Z$ satisfies

$$
\operatorname{rank}\left[\begin{array}{cc}
A & Y \\
X & Z
\end{array}\right]=\operatorname{rank} A
$$

if and only if

$$
Z=X A^{+} Y
$$

and thus $Z$ is a reflexive $g$-inverse of $A$.

(ii) Conversely, let $Z$ be any reflexive $g$-inverse of $A$. Then an $n \times n$ matrix $X$ and an $m \times m$ matrix $Y$ satisfy conditions (3.1), (3.2) and (3.3) if and only if

$$
X=Z A, \quad Y=A Z \text {. }
$$

(iii) In the above one-to-one correspondence between the pair $(X, Y)$ and $Z$, the matrix $Z$ is a minimum norm $g$-inverse of $A$ if and only if $X$ is symmetric. Similarly, $Z$ is a least squares g-inverse of $A$ if and only if $Y$ is symmetric.

Proof. Let rank $A=r$. Moreover, let $A$ and $B$ be partitioned as

$$
A=P\left[\begin{array}{cc}
A_{1} & O \\
O & O
\end{array}\right] Q^{\mathrm{T}}, \quad X=Q\left[\begin{array}{cc}
X_{1} & X_{2} \\
X_{3} & X_{4}
\end{array}\right] Q^{\mathrm{T}}
$$

where $P$ and $Q$ are orthogonal matrices and $A_{1}$ and $X_{1}$ are $r \times r$ matrices. We assume that (3.1) holds. Then noting that $A_{1}$ is invertible, we obtain from the condition $A X=A$ that $X_{1}=I$ and $X_{2}=O$. Furthermore, since rank $X=r, X_{4}$ must vanish. By applying the same procedure to $Y$, we know that conditions (3.1) and (3.2) hold if and only if $X$ and $Y$ are in the forms

$$
X=Q\left[\begin{array}{ll}
I_{r} & O \\
X_{3} & O
\end{array}\right] Q^{\mathrm{T}}, \quad Y=P\left[\begin{array}{cc}
I_{r} & Y_{2} \\
O & O
\end{array}\right] P^{\mathrm{T}}
$$

which implies that

$$
X A^{+} A=X, \quad A A^{+} Y=Y .
$$

Thus we have

$$
\left[\begin{array}{cc}
I & O \\
-X A^{+} & I
\end{array}\right]\left[\begin{array}{cc}
A & Y \\
X & Z
\end{array}\right]\left[\begin{array}{cc}
I & -A^{+} Y \\
O & I
\end{array}\right]=\left[\begin{array}{cc}
A & O \\
O & Z-X A^{+} Y
\end{array}\right] .
$$


Therefore, under assumption (3.1) and (3.2), we know that condition (3.3) holds if and only if $Z=X A^{+} Y$. In this case, from (3.1) and (3.2) we have $A Z A=A A^{+} A=A$ and $Z A Z=X A^{+} A A^{+} Y=Z$. This means $Z$ is a reflexive $g$-inverse of $A$. Thus we obtain (i).

We now prove (ii). It is easily verified that condition (3.5) gives (3.1), (3.2) and (3.3). To show the uniqueness, let $X$ and $Y$ be any matrices satisfying (3.1), (3.2) and (3.3). Then substituting (3.6) into (3.4), we have

$$
Z=Q\left[\begin{array}{cc}
A_{1}^{-1} & A_{1}^{-1} Y_{2} \\
X_{3} A_{1}^{-1} & X_{3} A_{1}^{-1} Y_{2}
\end{array}\right] P^{\mathrm{T}} .
$$

Hence, transformation (3.4) from the pair $(X, Y)$ to $Z$ is injective.

Finally, (iii) immediately comes from (3.5).

Remark 3.2. ¿From (3.6), we know that conditions (3.1) and (3.2) imply $X^{2}=$ $X$ and $Y^{2}=Y$. Hence, in the case where $Z=A^{+}$, the above theorem corresponds exactly to Theorem 1 in [2].

At the end of this paper, we shall state the following proposition in connection with Theorem A in [3].

Proposition 3.3. Let $A$ be any $m \times n$ matrix such that $\operatorname{rank} A=r$. Then we have the following claims.

(i) Let $R$ and $S$ be any $n \times(n-r)$ matrices that satisfy

$$
A R=O, \quad S^{\mathrm{T}} R \in G L(n-r) .
$$

We define an $n \times n$ matrix $X$ by

$$
X=I-R\left(S^{\mathrm{T}} R\right)^{-1} S^{\mathrm{T}} .
$$

Then $X$ satisfies condition (3.1). Conversely, any $n \times n$ matrix $X$ satisfying condition (3.1) is in the form (3.9).

(ii) Let $R$ and $S$ be any $m \times(m-r)$ matrices that satisfy

$$
A^{\mathrm{T}} R=O, \quad R^{\mathrm{T}} S \in G L(m-r) .
$$

We define an $m \times m$ matrix $Y$ by

$$
Y=I-S\left(R^{\mathrm{T}} S\right)^{-1} R^{\mathrm{T}} .
$$

Then $Y$ satisfies condition (3.2). Conversely, any $m \times m$ matrix $Y$ satisfying condition (3.2) is in the form (3.10).

Proof. We only prove (i), since (ii) is similarly proved. Let $X$ be defined by (3.9). Then from (3.8), we know $A X=A$. This means $\operatorname{rank} X \geq r$. On the other hand, we obtain $S^{\mathrm{T}} X=O$. Since $S^{\mathrm{T}} R$ is regular, $\operatorname{rank} S^{\mathrm{T}}=n-r$. This implies $\operatorname{rank} X \leq r$. Hence, $\operatorname{rank} X=r$ as desired.

Conversely, let $X$ be any $n \times n$ matrix satisfying condition (3.1). Furthermore, let $R$ be any $n \times(n-r)$ matrix such that $A R=O$ and $\operatorname{rank} R=n-r$. We put

$$
S=(I-X)^{\mathrm{T}} R .
$$


Then noting (3.7), we have

$$
\begin{aligned}
S^{\mathrm{T}} R & =R^{\mathrm{T}}(I-X) R \\
& =R^{\mathrm{T}} R-R^{\mathrm{T}} X A^{+} A R=R^{\mathrm{T}} R .
\end{aligned}
$$

Thus we know that $S^{\mathrm{T}} R \in G L(n-r)$. Moreover, from (3.1) and the assumption that $A R=O$, we have

$$
A\left(I-R\left(S^{\mathrm{T}} R\right)^{-1} S^{\mathrm{T}}-X\right)=O .
$$

On the other hand, from (3.12)

$$
\begin{aligned}
R^{\mathrm{T}}\left(I-R\left(S^{\mathrm{T}} R\right)^{-1} S^{\mathrm{T}}-X\right) & =R^{\mathrm{T}}\left(I-R\left(R^{\mathrm{T}} R\right)^{-1} R^{\mathrm{T}}(I-X)-X\right) \\
& =O
\end{aligned}
$$

Since

$$
\begin{aligned}
\operatorname{rank}\left[\begin{array}{ll}
A^{\mathrm{T}} & R
\end{array}\right]^{\mathrm{T}} & =\operatorname{rank}\left[\begin{array}{ll}
A^{\mathrm{T}} & R
\end{array}\right]^{\mathrm{T}}\left[\begin{array}{ll}
A^{\mathrm{T}} & R
\end{array}\right] \\
& =\operatorname{rank} A+\operatorname{rank} R=n
\end{aligned}
$$

equations (3.13) and (3.14) mean $I-R\left(S^{\mathrm{T}} R\right)^{-1} S^{\mathrm{T}}-X=O$. This gives the proof. $\square$

REMARK 3.4. In (3.11), if $X$ is symmetric, then we have $A S=A(I-X) R=O$.

This means $\left[\begin{array}{ll}A^{\mathrm{T}} & R\end{array}\right]^{\mathrm{T}}(S-R)=O$. Thus we have $S=R$. The same is true for claim (ii).

\section{REFERENCES}

[1] Thomas L. Boullion and Patrick L. Odell. Generalized Inverse Matrices. Wiley-Interscience, New York, 1971.

[2] Miroslav Fiedler and Thomas L. Markham. A characterization of the Moore-Penrose inverse. Linear Algebra and its Applications, 179:129-133, 1993.

[3] Miroslav Fiedler. Moore-Penrose biorthogonal systems in Euclidean spaces. Linear Algebra and its Applications, 362:137-143, 2003. 